\title{
The Longitudinal Trajectory of Default Mode Network Connectivity in Healthy Older Adults Varies As a Function of Age and Is Associated with Changes in Episodic Memory and Processing Speed
}

\author{
@Adam M. Staffaroni, ${ }^{1}$ Jesse A. Brown, ${ }^{1}$ Kaitlin B. Casaletto, ${ }^{1}$ Fanny M. Elahi, ${ }^{1}$ Jersey Deng, ${ }^{1}$ John Neuhaus, ${ }^{3}$ \\ Yann Cobigo, ${ }^{1}$ @Paige S. Mumford, ${ }^{1}$ Samantha Walters, ${ }^{1}$ - Rowan Saloner, ${ }^{1}$ Anna Karydas, ${ }^{1}{ }^{\mathbb{C}}$ Giovanni Coppola, ${ }^{4}$ \\ Howie J. Rosen, ${ }^{1}$ Bruce L. Miller, ${ }^{1}$ William W. Seeley, ${ }^{1,2}$ and Joel H. Kramer ${ }^{1}$ \\ Memory and Aging Center, Departments of ${ }^{1}$ Neurology, ${ }^{2}$ Pathology, ${ }^{3}$ Department of Epidemiology and Biostatistics, University of California, San Francisco, \\ San Francisco, California 94143, and ${ }^{4}$ Departments of Psychiatry and Neurology, David Geffen School of Medicine, University of California Los Angeles, Los \\ Angeles, California 90095
}

The default mode network (DMN) supports memory functioning and may be sensitive to preclinical Alzheimer's pathology. Little is known, however, about the longitudinal trajectory of this network's intrinsic functional connectivity (FC). In this study, we evaluated longitudinal FC in 111 cognitively normal older human adults (ages 49-87, 46 women/65 men), 92 of whom had at least three task-free fMRI scans ( $n=353$ total scans). Whole-brain FC and three DMN subnetworks were assessed: (1) within-DMN, (2) between anterior and posterior DMN, and (3) between medial temporal lobe network and posterior DMN. Linear mixed-effects models demonstrated significant baseline age $\times$ time interactions, indicating a nonlinear trajectory. There was a trend toward increasing FC between ages $50-66$ and significantly accelerating declines after age 74. A similar interaction was observed for whole-brain FC. APOE status did not predict baseline connectivity or change in connectivity. After adjusting for network volume, changes in within-DMN connectivity were specifically associated with changes in episodic memory and processing speed but not working memory or executive functions. The relationship with processing speed was attenuated after covarying for white matter hyperintensities (WMH) and whole-brain FC, whereas withinDMN connectivity remained associated with memory above and beyond WMH and whole-brain FC. Whole-brain and DMN FC exhibit a nonlinear trajectory, with more rapid declines in older age and possibly increases in connectivity early in the aging process. Within-DMN connectivity is a marker of episodic memory performance even among cognitively healthy older adults.

Key words: aging; cognition; default mode network; episodic memory; functional MRI; neuroimaging

Significance Statement

Default mode network and whole-brain connectivity, measured using task-free fMRI, changed nonlinearly as a function of age, with some suggestion of early increases in connectivity. For the first time, longitudinal changes in DMN connectivity were shown to correlate with changes in episodic memory, whereas volume changes in relevant brain regions did not. This relationship was not accounted for by white matter hyperintensities or mean whole-brain connectivity. Functional connectivity may be an early biomarker of changes in aging but should be used with caution given its nonmonotonic nature, which could complicate interpretation. Future studies investigating longitudinal network changes should consider whole-brain changes in connectivity.

\section{Introduction}

The default mode network (DMN) is an important network in aging, as it is selectively vulnerable to the earliest stages of Alzheimer's disease (AD) pathology (Greicius et al., 2004; Hedden et al.,
2009; Sheline et al., 2010b), which accumulates years before clinical manifestations (Braak and Del Tredici, 2012). Although cross-sectional task-free (resting state) functional MRI (tf-fMRI) studies have suggested that the functional connectivity (FC) of
P.S.M., and S.W. analyzed data; A.M.S., J.A.B., K.B.C., F.M.E., J.N., Y.C., P.S.M., R.S., H.J.R., B.L.M., W.W.S., and J.H.K.
wrote the paper.
This work was supported by the NIH-NIA (R01AG032289, R01AG048234, UCSF ADRC P50 AG023501) and the

Larry L. Hillblom Network Grant for the Prevention of Age-Associated Cognitive Decline (2014-A-004-NET). 
the brain changes with age and APOE gene status and correlates with cognition (Sala-Llonch et al., 2015), there are a paucity of studies investigating how these relationships evolve within subjects over time. Understanding the normative longitudinal trajectory of network connectivity in healthy older adults is important to both elucidate the biology associated with "normal" brain aging and to study its role as a biomarker in patient populations (Barkhof et al., 2014).

Most cross-sectional studies suggest that older subjects have reduced DMN FC compared with younger adults (AndrewsHanna et al., 2007; Damoiseaux et al., 2008; Sala-Llonch et al., 2015). Granular investigations of the DMN subsystems, however, indicate that some DMN components may in fact show increased FC with age and AD pathology (Jones et al., 2011; Damoiseaux et al., 2012; Li et al., 2016). FC between anterior and posterior portions of the DMN (apDMN), as well as the connectivity between the medial temporal lobe and cortical portions of the DMN (MTL-pDMN), may be most affected by aging (Andrews-Hanna et al., 2007) and AD pathology (Jones et al., 2016). Few longitudinal DMN investigations exist, and most have examined only two time points and are therefore unable to model nonlinear effects or control for regression to the mean. Two studies found between-subject, cross-sectional effects of age but not withinsubject changes in DMN connectivity (Persson et al., 2014; Fjell et al., 2015). Ng et al. (2016) observed that within-DMN FC declined over time .

The APOE $\varepsilon 4$ allele is a well established $\mathrm{AD}$ genetic risk factor (Corder et al., 1993), which may affect FC across the lifespan. In two of three studies, adult $\varepsilon 4$ carriers in their 20 s and early 30 s exhibited increased within-DMN connectivity (Filippini et al., 2009) and increased connectivity between the hippocampi and cortical regions (Zheng et al., 2017), but conflicting results have been reported (Su et al., 2017). Studies in older adults also suggest that there is a relationship between APOE genotype and FC, although the exact nature of this relationship appears complicated with variable findings reported in the literature (Sheline et al., 2010a; Machulda et al., 2011; Westlye et al., 2011; Jones et al., 2016).

Cross-sectional research has demonstrated that decreased connectivity within the DMN and apDMN is associated with poorer memory performance (Andrews-Hanna et al., 2007; Vidal-Piñeiro et al., 2014; Sala-Llonch et al., 2015; Ward et al., 2015). Longitudinal studies have demonstrated that memory declines are linked to decreased connectivity in the DMN, particularly the posterior cingulate cortex (PCC) (Persson et al., 2014; Bernard et al., 2015). Fjell et al. (2015) showed a dissociation between the relationship of connectivity and memory as a function of age. Older participants (ages 63-86) showed the expected positive correlation between memory and DMN connectivity, whereas the younger cohort (ages 23-52) showed an inverse relationship, such that increased connectivity correlated with poorer memory performance. Together, DMN connectivity is associated with memory but the relationship is nuanced and may differ across the lifespan.

We sought to characterize baseline differences and longitudinal trajectories of DMN subsystems in adults ages 49-89. Parcellating the DMN into subsystems appears to be critical for understanding the nuanced effects of aging this network. In ad-

The authors declare no competing financial interests.

Correspondence should be addressed to Dr. Adam Staffaroni, Memory and Aging Center, Department of Neurology, University of California, San Francisco, 675 Nelson Rising Lane, Suite 19, San Francisco, CA 94143. E-mail: Adam.Staffaroni@ucsf.edu.

DOI:10.1523/JNEUROSCI.3067-17.2018

Copyright $\odot 2018$ the authors $\quad 0270-6474 / 18 / 382810-09 \$ 15.00 / 0$ dition to measuring FC of the whole DMN, we analyzed apDMN, and MTL-pDMN. We also analyzed the effect of APOE status on baseline and longitudinal trajectory, and examined the relationship between DMN and cognition.

\section{Materials and Methods}

\section{Participants}

Participants were recruited at the University of California, San Francisco's (UCSF) Memory and Aging Center as part of the Hillblom Healthy Aging Study, a deeply phenotyped longitudinal cohort. Visits included neuropsychological testing and a neurologic examination in addition to an MRI scan. The neurologic examination was used to confirm participants' status as clinically normal. From this cohort, we initially included all participants with at least three time points of tf-fMRI acquisition (371 scans). Two participants who were diagnosed with mild cognitive impairment and another who developed Parkinson's disease were excluded. After excluding for excessive head motion as described below, the dataset comprised 111 participants with a total of 353 scans for an average of 3.2 observations per person (range: 1-7); 108 had at least two time points, 92 had at least three time points. The average interscan interval was 2 years (SD: 1.1$)$ with a wide range (0.4-7.7 years). Mean age was 69.3 (SD: 6.7) with a range of $49-87$. Mean education was 17.8 years (SD: 2.0$)$ with a range of 12-20. Forty-six participants identified as female. Mean MMSE score was 29.4 (SD: 0.8; median: 30) with a range of 27-30. Of the 111 participants included in this study, only 8 were lost to attrition. Reasons for withdrawal ranged from travel inconveniences to serious medical illnesses. We reran the analyses without these eight participants, as a sensitivity analysis, and the magnitude of the associations were very similar.

\section{Cognitive measures}

Composite measures were used to summarize neuropsychological performance on several paper and pencil and computerized measures. $Z$-scores were calculated for individual tests and then averaged across the subtests that were included in the composite. An episodic memory composite score was created using a measure of visual memory, Benson Figure Recall (Kramer et al., 2003), and the California Verbal Learning Test, second edition (CVLT-II; Delis et al., 2000) subscores: immediate recall total, long (20 min) delay free recall total, and recognition discriminability $\left(\mathrm{d}^{\prime}\right)$. We also created composites that summarized performance in the domains of processing speed, working memory, and executive functioning to evaluate the specificity of the relationship between DMN FC and episodic memory. The working memory composite comprised digit span backward (Wechsler, 1997), as well as several computerized measures of working memory, including dot counting (Kramer et al., 2014), n-back (1- and 2-back), and running letter memory. The executive functions composite included Stroop interference (Stroop, 1935), modified trail making test (Kramer et al., 2003), phonemic fluency (number of D-words/min; Kramer et al., 2003), and design fluency (DKEFS Condition 1; Delis et al., 2001). Finally, the processing speed composite summarizes performance on six visuospatial processing speed tasks described in detail by Kerchner et al. (2012). The composite created here does not include the mental rotation task, which was excluded because this test is no longer administered and therefore would be missing at many observations.

\section{Neuroimaging}

Scanner information. Subjects were scanned at the UCSF Neuroscience Imaging Center on a Siemens Trio 3T scanner. A T1-weighted MP-RAGE structural scan was acquired with an acquisition time $=8 \min 53 \mathrm{~s}$, sagittal orientation, a field-of-view of $160 \times 240 \times 256 \mathrm{~mm}$ with an isotropic voxel resolution of $1 \mathrm{~mm}^{3}$, TR $=2300 \mathrm{~ms}, \mathrm{TE}=2.98 \mathrm{~ms}$, TI $=$ $900 \mathrm{~ms}$, flip angle $=9^{\circ}$. Task-free T2*-weighted echoplanar fMRI scans were acquired with an acquisition time $=8 \min 06 \mathrm{~s}$, axial orientation with interleaved ordering, field-of-view $=230 \times 230 \times 129 \mathrm{~mm}$, matrix size $=92 \times 92$, effective voxel resolution $=2.5 \times 2.5 \times 3.0 \mathrm{~mm}, \mathrm{TR}=$ $2000 \mathrm{~ms}, \mathrm{TE}=27 \mathrm{~ms}$, for a total of 240 volumes. During the $8 \mathrm{~min}$ tf-fMRI acquisition protocol, participants were asked to close their eyes and concentrate on their breath.

Longitudinal T1 processing. Before preprocessing, all T1-weighted images were visually inspected for quality control. Images with excessive 

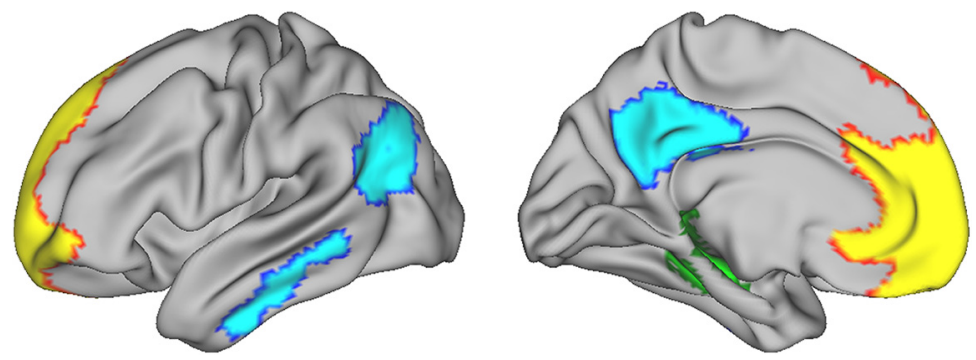

Figure 1. DMN and medial temporal lobe subsystems. Anterior (yellow), posterior (blue), and medial temporal lobe (green) subsystems derived from a data-driven network parcellation.

motion or image artifact were excluded. T1-weighted images underwent bias field correction using N3 algorithm, and segmentation was performed using SPM12 (Wellcome Trust Center for Neuroimaging, London, UK; http://www.fil.ion.ucl.ac.uk/spm; RRID:SCR_007037) unified segmentation (Ashburner and Friston, 2005). An intrasubject template was created by nonlinear diffeomorphic and rigid-body registration proposed by the symmetric diffeomorphic registration for longitudinal MRI framework (Ashburner and Ridgway, 2012). The intrasubject template was also segmented using SPM12's unified segmentation. A group template was generated from the within-subject average gray and white matter tissues by nonlinear and rigid-body registration template generation using Diffeomorphic Anatomical Registration using Exponentiated Lie algebra (DARTEL; Ashburner, 2007). Native subjects' space gray and white matter were normalized, modulated, and smoothed in the group template using intrasubject and intersubject transformations. The applied smoothing used a Gaussian kernel with $\sim 4 \mathrm{~mm}$ full-width half-maximum. For statistical purposes, linear and nonlinear transformations between DARTEL's space and International Consortium for Brain Mapping (Mazziotta et al., 2001) was applied. Each subject's average subject segmentations were carefully inspected to ensure no major segmentation or normalization errors.

fMRI preprocessing. For each fMRI scan, the first five volumes were discarded. SPM12 RRID:SCR_002823 software was used for subsequent fMRI preprocessing. The remaining 235 volumes were slice-time corrected, realigned to the mean functional image, and assessed for rotational and translational head motion. Volumes were next coregistered to the MP-RAGE image, then normalized to the standard MNI-152 healthy adult brain template using SPM segment, producing MNI-registered volumes with $2 \mathrm{~mm}^{3}$ isotropic resolution. These volumes were spatially smoothed with a 6 $\mathrm{mm}$ radius Gaussian kernel and temporally bandpass filtered in the $0.008-0.15 \mathrm{~Hz}$ frequency range using fslmaths. Nuisance parameters in the preprocessed data were estimated for the CSF using a mask in the central portion of the lateral ventricles and for the white matter using a mask of the highest probability cortical white matter as labeled in the FSL tissue prior mask. Additional nuisance parameters included the three translational and three rotational motion parameters, the temporal derivatives of the previous eight terms (WM/CSF/6 motion), and the squares of the previous 16 terms (Satterthwaite et al., 2013). Subjects were included only if they met all of the following criteria: no inter-frame head translations $>3 \mathrm{~mm}$, no inter-frame head rotations $>3^{\circ}$, and $<24$ motion spikes (defined as inter-frame head displacements $>1 \mathrm{~mm}), 10 \%$ of the total number of frames.

Process of excluding nodes. We excluded regions with insufficient fMRI BOLD signal-to-noise ratio by first calculating each region's mean BOLD intensity across all 371 scans. For a given region, we identified all scans where signal was within $2.5 \%$ of the minimum BOLD intensity. If three or fewer scans met this criterion, we excluded those scans from subsequent analysis on the basis that they were outliers. Otherwise, we partitioned the scans into two groups, those with signal less than the $2.5 \%$ cutoff and those with signal greater than the $2.5 \%$ cutoff. We then performed a two-sample $t$ test on the BOLD intensity values for these two groups. If that $t$ statistic was $t<-2.3$, we excluded the region, based on the rationale that this node had significantly reduced signal in a substantial portion of scans. Based on this procedure, we dropped 49 scans and
45 nodes. Those nodes that were part of the DMN or hippocampal networks were as follows: left frontal medial cortex (47), right frontal pole (48), left inferior (95), and middle (81) temporal gyri, posterior divisions, and several left and right parahippocampal nodes (109, $110,111,115,116$, and 117). Numbers in parentheses correspond to the nodes in the Brainnetome atlas (Fan et al., 2016).

Network construction. We defined the DMN using a set of 75 age-matched healthy older control subjects (our "HC2" group; mean age $=65.3 \pm 10.0$ years, 33 females $/ 42$ males, mean education $=17.3 \pm 2.1$ years, 68 right handed/7 left handed) scanned and analyzed using the same pipeline as the subjects in the longitudinal portion of this study. Twenty-seven of 75 of the HC2 controls were also included in the primary analysis. In HC2 subjects, we determined the whole-brain functional connectome using 228 regions from the Brainnetome atlas (Fan et al., 2016). Network connectivity was determined by applying the 228 regional masks to a given subject's preprocessed tf-fMRI data to extract regional mean time series, and partially correlating those time series in a pairwise fashion again controlling for the 32 nuisance parameters to obtain the $228 \times 228$ covariate-adjusted functional connectivity matrix. Each matrix was then $r$-to- $z$ transformed. These matrices were averaged to produce the HC2 group average functional connectome. We then applied a modularity-based method for identifying which nodes comprised each module or "intrinsic connectivity network", including the DMN, adopting a conceptually similar strategy similar to that used by Power et al. (2011). The HC2 network was analyzed with the Brain Connectivity Toolbox (https://sites.google.com/ site/bctnet/). The modular structure of the network was determined by calculating the Louvain modularity (gamma $=1$ ) on the un-thresholded group matrix 1000 times and finding the partition that balanced the criteria of being among the most frequent solutions and maximizing the modularity value of $Q$, the quality of the partitioning (Rubinov and Sporns, 2011). We labeled the winning partition as the fixed "community index", which yielded four modules. We then recursively applied this algorithm a second time within each module to yield a total of 15 submodules. Visual inspection unambiguously revealed networks corresponding to the DMN, containing 18 Brainnetome regions, along with the other canonical intrinsic connectivity networks (Fig. 1). A third recursive application of the modularity algorithm yielded 32 submodules and segmented the DMN into an anterior component including the medial prefrontal cortex and anterior cingulate, and a posterior component including the precuneus, posterior cingulate, angular gyrus, and middle/ inferior lateral temporal lobe. These two DMN partitions closely correspond to the anterior and posterior DMN described by Damoiseaux et al. (2012).

The cortical DMN network included anterior regions (anterior cingulate gyrus, paracingulate gyrus, medial frontal cortex, and areas of the frontal pole) and posterior regions [PCC, precuneus, angular gyrus, and posterior regions of the middle and inferior temporal gyrus]. A separate medial temporal lobe network was created that included hippocampal and parahippocampal nodes, as well as connection to the retrosplenial cortex. Both the DMN and medial temporal lobe parcellations closely recapitulate the parcels described by Andrews-Hanna et al. (2010). We calculated three mean functional connectivity values by taking the mean of the edges between all nodes within or between networks: (1) the cortical DMN, (2) apDMN, and (3) MTL-pDMN. We decided to use the term "posterior DMN", as we feel it is intuitive and includes all of the commonly included posterior aspects of the DMN: precuneus and PCC. Common nomenclature has not been established, with some groups using terms such as ventral to describe a DMN parcellation that overlaps with the posterior regions describe above. Whole-brain FC was calculated by taking the mean of the edges between all nodes in the brain that were not excluded.

The Brainnetome regions comprising the tf-fMRI networks (anterior $\mathrm{DMN}$, posterior $\mathrm{DMN}$, and medial temporal lobe) were then 
applied to the T1 scans to extract gray matter volumes. The regional volumes for all regions comprising a given network were summed (and divided by total intracranial volume) to obtain the network's gray matter volume.

White matter hyperintensity quantification. White matter hyperintensities (WMH) were segmented using FLAIR and T1-weighted images. We visually inspected the raw scans for quality control. The WMH segmentation process is fully automated and based on a regression algorithm (Dadar et al., 2017) and using an Hidden Markov Random Field with Expectation Maximization software (Avants et al., 2011). Every segmentation was visually assessed for accuracy.

\section{APOE genotyping and demographics}

Genomic DNA was extracted from peripheral blood using standard protocols (Gentra PureGene Blood Kit, Qiagen). Genotyping was performed using either TaqMan or Sequenom genotyping. TaqMan Allelic Discrimination Assay was used for APOE genotyping (rs429358 and rs7412), and was conducted on an ABI 7900HT Fast Real-Time PCR system (Applied Biosystems) according to the manufacturer's instructions.

The SpectroAquire and MassARRAY Typer Software packages (Sequenom) were used for interpretation and Typer analyzer (v3.4.0.18) was used to review and analyze data. 81 participants with 258 scans were classified as non-carriers and 30 participants with 95 scans were $\varepsilon 4$ carriers. Mean age for the carriers was 68.0 (6.1) with a range of 55-81.

\section{Experimental design and statistical analysis}

We assessed the longitudinal relationship between age and changes in DMN volume by constructing linear mixed effects (LME) models with a time (in years) by baseline age (time invariant; centered at mean) interaction term and main effects for time and baseline age. Time was entered as a continuous variable. Gender was entered as a nuisance covariate. All LME analyses were modeled with random slopes and intercepts. In those models that failed to estimate SEs or failed to converge, random intercept only models were run. Unstandardized regression coefficients $(b)$ are reported for LME models. The main effects for time in those models with an interaction term are interpreted as the slope for participants of mean age. All statistical analyses were performed in Stata 14.2 (StataCorp, 2015).

We next assessed the relationship between connectivity and baseline age in all three networks of interest (within-DMN, apDMN, and MTLpDMN), as well as whole-brain connectivity, again using LME models with a time by baseline age (time invariant) interaction term and main effects for time and baseline age. Gender was again entered as a covariate. We chose not to control for motion at the group level based on recent work suggesting that doing so can remove true, age-related connectivity effects (Geerligs et al., 2017). We reran all analyses covarying for total displacement and found the same pattern of results.

Baseline differences in connectivity were assessed using linear regression with $A P O E$ status as a dummy-coded variable controlling for age and gender. Participants were considered to be $\varepsilon 4$ carriers whether they were homozygotes or heterozygotes. To assess for differences in rates of DMN change, we used a LME model with a time $\times$ APOE interaction for all three FC metrics.

Finally, LME models were used to assess the correlation between change in FC (time-varying predictor) and change in cognition $(n=94)$. In these models, baseline age, education, and gender were entered as covariates. FC estimates were decomposed into between- and withinperson variance decomposed by calculating the mean FC for each subject (between-person) and then subtracting each person's mean from their FC estimate (within-person); these were entered as independent predictors. This approach clarifies whether intraindividual or interindividual differences in FC are associated with cognition. We then added other covariates to the model in a stepwise fashion: regional volume and mean whole-brain FC. Because there was a smaller sample with successful WMH segmentation, we ran those analyses separately, with whole-brain FC in the model $(n=88)$.

In a post hoc analysis to provide context to our results, we assessed longitudinal changes in the cognitive scores by fitting four mixed effects models with cognition as the outcome, a time $\times$ age interaction, and main effects for time and age.

\section{Results}

\section{Cortical DMN volume}

DMN volume showed a significant, negative interaction between baseline age and change over time $\left(b=-6.2 \times 10^{-6}, 95 \% \mathrm{CI}\right.$ : $\left.-1.1 \times 10^{-5},-1.7 \times 10^{-6} ; p=0.006\right)$, as well as a significant main effect of time $\left(b=-1.3 \times 10^{-4}, 95 \% \mathrm{CI}:-1.5 \times 10^{-4}\right.$, $\left.-1.1 \times 10^{-4} ; p<0.001\right)$ and age $\left(b=-8.6 \times 10^{-5}, 95 \% \mathrm{CI}\right.$ : $\left.-1.5 \times 10^{-4},-2.1 \times 10^{-5} ; p=0.01\right)$. This indicates that DMN volume declined over time, was lower with older age, and the older the individual, the faster their rate of decline. Predicted values are plotted in Figure 2-1 and suggest a sharper rate of decline with increasing age.

\section{Intraindividual trajectories of DMN connectivity change as a function of age \\ Within-DMN connectivity}

Longitudinal intraindividual change in DMN FC showed a significant, negative interaction with baseline age $(b=-9.7 \times$ $\left.10^{-4}, 95 \% \mathrm{CI}:-1.7 \times 10^{-3},-1.5 \times 10^{-4} ; p=0.020\right)$; the main effect for time at mean baseline age was not significant $(b=$ $\left.-3.1 \times 10^{-3}, 95 \% \mathrm{CI}:-7.9 \times 10^{-3}, 1.5 \times 10^{-3} ; p=0.185\right)$. This interaction remained significant after controlling for $\mathrm{WMH}$ $\left(b=-1.5 \times 10^{-3}, 95 \% \mathrm{CI}:-2.8 \times 10^{-3},-3.5 \times 10^{-4} ; p=\right.$ 0.011). The relationship between baseline age and FC was not significant $\left(b=1.6 \times 10^{-3}, 95 \% \mathrm{CI}:-9.8 \times 10^{-4}, 4.2 \times 10^{-3}\right.$; $p=0.222)$. Change in DMN volume was positively associated with changes in DMN FC $(b=8.3,95 \%$ CI: $1.9,14.7 ; p=0.011)$. The intercept-slope covariance was positive but nonsignificant $\left(2.2 \times 10^{-4}, 95 \% \mathrm{CI}:-5.2 \times 10^{-5}, 5.0 \times 10^{-4}\right)$; a positive covariance indicates that those with lower $\mathrm{FC}$ at baseline decline in FC at a faster rate and vice versa.

Plotting the fitted values for the interaction model for each individual revealed a nonlinear curve (Fig. 2-2), and plots of the predicted slopes at different ages (Fig. 2-3) indicated that the interaction effect was driven by increasing DMN connectivity in younger participants, flatting of the slope at approximately age 70 , and increasingly steeper declines in DMN connectivity as participants aged beyond 70 .

To further understand this effect, we divided the sample into three age groups: $(1)<66(n=33)$, (2) 66-74 $(n=55)$, and (3) $>74(n=23)$. We fitted separate models for each age group with time as the predictor of interest and FC as the outcome. The youngest participants showed a positive, but nonsignificant slope $\left(b=6.6 \times 10^{-3}, 95 \% \mathrm{CI}:-2.9 \times 10^{-3}, 1.6 \times 10^{-2} ; p=0.175\right)$. Those between the ages of 66 and 74 showed a trend toward a negative slope $\left(b=-5.2 \times 10^{-3}, 95 \% \mathrm{CI}:-1.1 \times 10^{-2}, 9.8 \times\right.$ $\left.10^{-4} ; p=0.097\right)$, whereas those over the age of 74 showed significant declines $\left(b=-1.3 \times 10^{-2}, 95 \% \mathrm{CI}:-2.4 \times 10^{-2}\right.$, $\left.-1.1 \times 10^{-3} ; p=0.032\right)$. This suggests that the age $\times$ time interaction is primarily driven by declining FC in the oldest adults. Raw subject-specific trajectories, color coded by age group, are displayed in Figure 3.

\section{Anterior-posterior DMN}

There was a significant age $\times$ time interaction for anterior to posterior DMN FC $\left(b=-1.2 \times 10^{-3}, 95 \% \mathrm{CI}:-2.2 \times 10^{-3}\right.$, $\left.-7.4 \times 10^{-5} ; p=0.036\right)$. The main effect of baseline age $(b=$ $\left.1.3 \times 10^{-3}, 95 \% \mathrm{CI}:-2.3 \times 10^{-3}, 4.9 \times 10^{-3} ; p=0.484\right)$ and time $\left(b=-5.2 \times 10^{-3}, 95 \% \mathrm{CI}:-1.1 \times 10^{-2}, 9.8 \times 10^{-4} ; p=\right.$ $0.098)$ were not significant. The interaction effect remained significant when DMN volume was covaried $\left(b=-1.1 \times 10^{-3}\right.$, $95 \%$ CI: $\left.-2.2 \times 10^{-3},-3.0 \times 10^{-5} ; p=0.044\right)$. 
2-1.

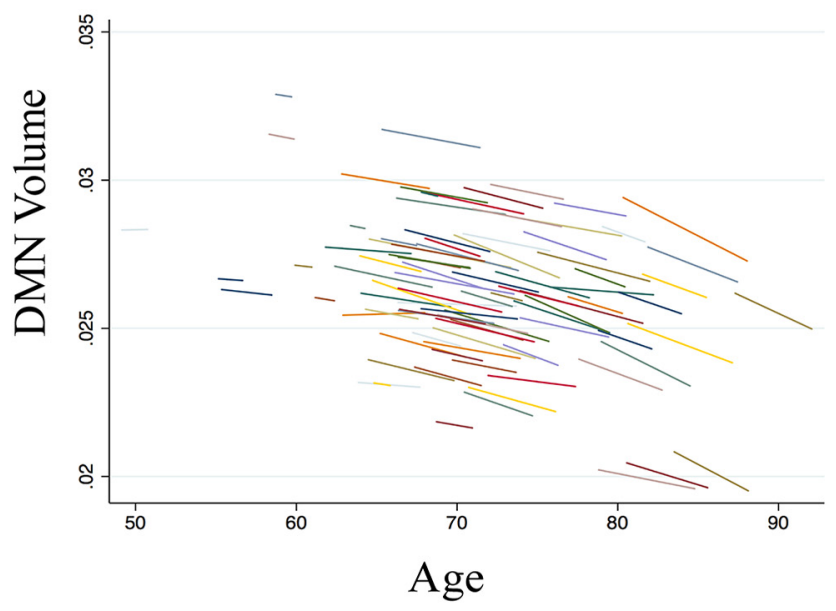

2-2.

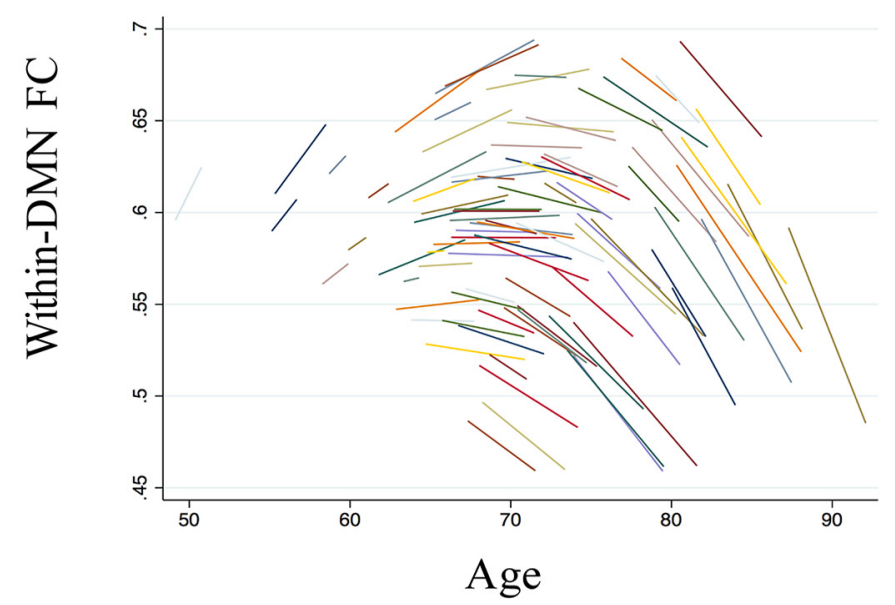

\section{2-3.}

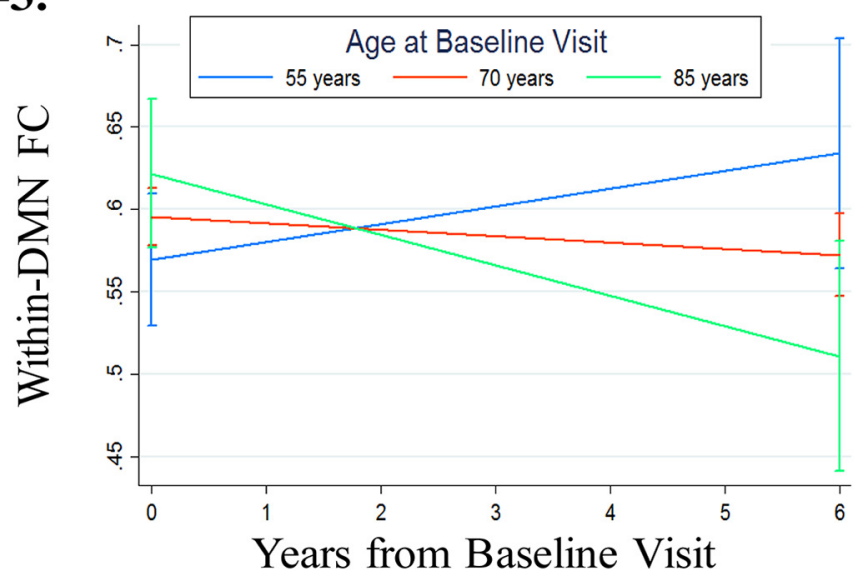

Figure 2. Baseline age $\times$ time interactions of DMN volume and FC. 2-1 and 2-2, Fitted values from linear mixed-effects models with random slope and intercepts are plotted for (2-1) DMN volume (controlling for total intracranial volume) and (2-2) mean within-DMN FC. Age is shown on the $x$-axis and fitted values for the outcome of interest are displayed on the $y$-axis. Each line represents an individual participant's fitted trajectory. 2-3, The predicted slopes of within-DMN FC at three values of baseline age.

Posterior DMN and medial temporal lobe coupling

Neither the baseline age $\times$ time interaction $\left(b=-5.6 \times 10^{-4}\right.$, $95 \%$ CI: $\left.-1.6 \times 10^{-3}, 5.1 \times 10^{-4} ; p=0.306\right)$ or main effects of baseline age $\left(b=9.1 \times 10^{-4}, 95 \% \mathrm{CI}:-2.8 \times 10^{-3}, 4.6 \times 10^{-3}\right.$; $p=0.635)$ or time $\left(b=-3.5 \times 10^{-3}, 95 \% \mathrm{CI}:-9.8 \times 10^{-3}\right.$, $\left.2.7 \times 10^{-3} ; p=0.306\right)$ were significant.

\section{Whole-brain connectivity}

Longitudinal whole-brain FC changes were strongly associated with longitudinal within-DMN FC ( $b=0.61,95 \%$ CI: $0.55,0.67$; $p<0.001)$. Similar to within-DMN connectivity, there was a significant age $\times$ time interaction for whole-brain FC $(b=$ $\left.-1.1 \times 10^{-3}, 95 \% \mathrm{CI}:-2.0 \times 10^{-3},-1.9 \times 10^{-4} ; p=0.018\right)$. Main effects of baseline age $\left(b=1.7 \times 10^{-3}, 95 \% \mathrm{CI}:-1.8 \times\right.$ $\left.10^{-3}, 5.1 \times 10^{-3} ; p=0.340\right)$ and time $\left(b=-1.6 \times 10^{-3}, 95 \%\right.$ CI: $\left.-6.9 \times 10^{-3}, 3.7 \times 10^{-3} ; p=0.550\right)$ were not significant. Similar to within-DMN connectivity, the interaction appears to be driven by faster decline in the oldest adults in this sample. When rates of change where analyzed separately in the three age groups defined above, significant decline was observed in those $>74\left(b=-1.4 \times 10^{-2}, 95 \% \mathrm{CI}:-2.6 \times 10^{-2},-1.0 \times 10^{-3} ; p=\right.$ $0.034)$. Nonsignificant decline was seen in the $66-74$ group $(b=$ $\left.-1.4 \times 10^{-3}, 95 \% \mathrm{CI}:-8.5 \times 10^{-3}, 5.7 \times 10^{-3} ; p=0.695\right)$ and nonsignificant increases were seen in those ages $<66(b=5.7 \times$ $\left.10^{-3}, 95 \% \mathrm{CI}:-5.1 \times 10^{-3}, 1.7 \times 10^{-2} ; p=0.229\right)$.

\section{The relationship between changes in connectivity and APOE status}

$A P O E \varepsilon 4$ carriers and non-carriers did not differ significantly on baseline FC ( $p=0.455-0.958)$ or rate of change in FC ( $p=$ $0.127-0.969$ ) for any of the three DMN metrics after controlling for age and gender.

\section{Intraindividual changes in DMN connectivity are specifically associated with changes in episodic memory}

Within-DMN connectivity and memory

Overall, mean FC levels were not correlated with memory performance when we examined FC of the DMN $(b=0.71,95 \% \mathrm{CI}$ : $-0.7,2.1 ; p=0.316), \operatorname{apDMN}(b=0.45,95 \% \mathrm{CI}:-0.6,1.5 ; p=$ $0.398)$, or pDMN-MTL $(b=0.78,95 \% \mathrm{CI}:-2.6,1.8 ; p=0.142)$.

Within-person changes in within-DMN connectivity, however, were positively associated with changes in episodic memory $(b=0.71,95 \%$ CI: $0.1,1.4 ; p=0.034)$. This relationship remained significant after controlling for atrophy in the DMN $(b=$ $0.72,95 \%$ CI: $0.1,1.4 ; p=0.031)$, and when volume of the MTL 


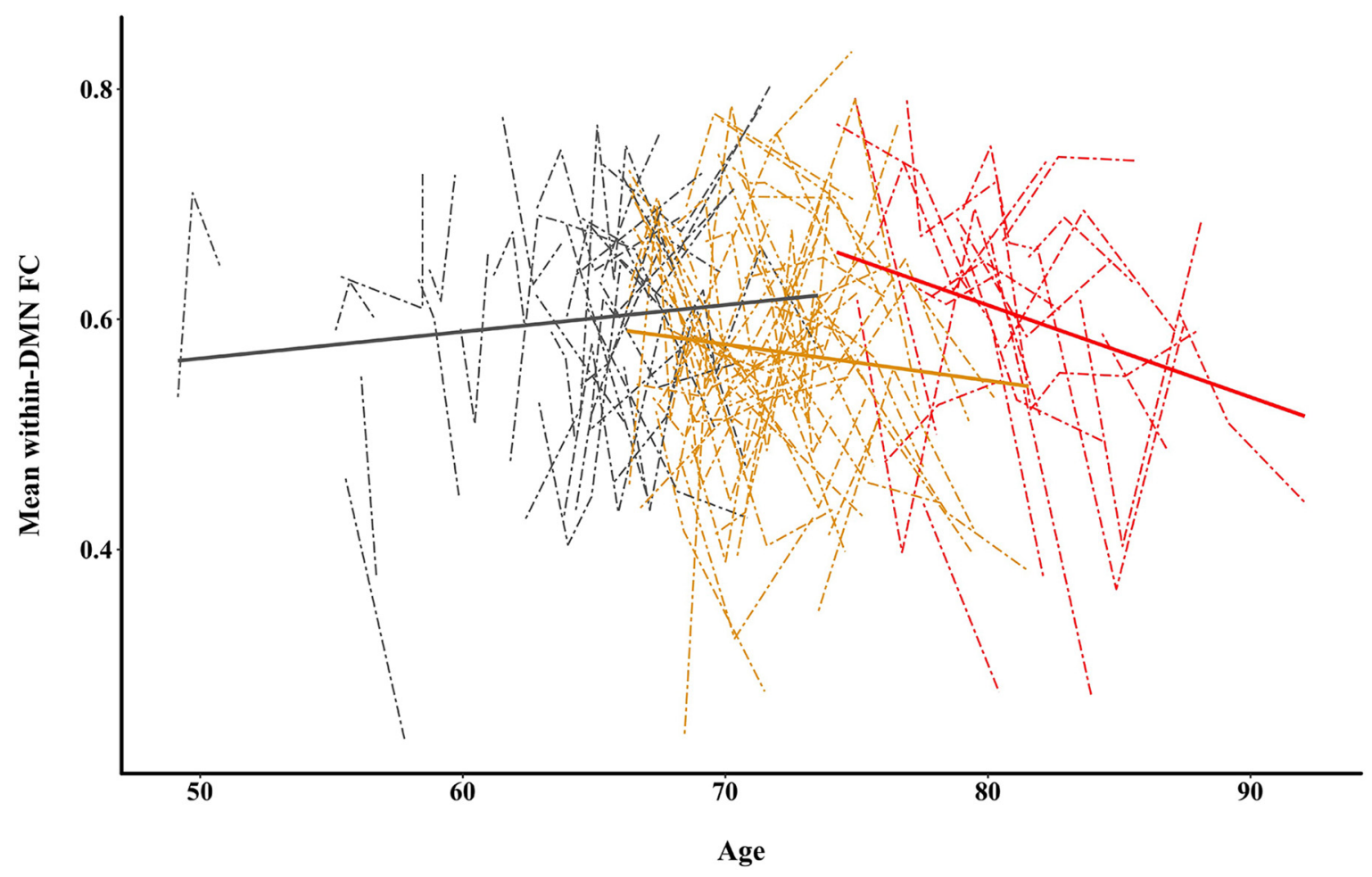

Figure 3. Subject-specific trajectories of raw within-DMN FC. Raw subject-specific trajectories for each participant are presented using dashed lines. Coloring is used to indicate participant's baseline age. Solid lines display fitted regression lines for each age cohort.

network was added as an additional covariate $(b=0.83,95 \% \mathrm{CI}$ : $0.2,1.5 ; p=0.010)$. Notably, in these same models, neither atrophy in the cortical DMN $(b=-32.4,95 \% \mathrm{CI}:-97.8,33.0 ; p=$ $0.332)$ nor the MTL networks $(b=-66.7,95 \%$ CI: -477.5 , $344.1 ; p=0.750)$ were significantly associated with memory performance above and beyond FC. The parameter was increased slightly when whole-brain connectivity was added to the model $\left(b=0.84,95 \% \mathrm{CI}:-1.4 \times 10^{-2}, 1.7 ; p=0.059\right)$; whole-brain connectivity was not associated with memory $\left(b=-9.1 \times 10^{-3}\right.$, 95\% CI: $-0.8,0.7 ; p=0.981)$. Notably, within-DMN connectivity remained associated with episodic memory even after controlling for white matter hyperintensities and mean FC ( $b=1.2,95 \%$ CI: $0.1,2.3 ; p=0.037$ ).

There was a trend for a relationship between memory and the apDMN FC $(b=0.43,95 \%$ CI: $-0.1,0.9 ; p=0.083)$, which became significant after controlling for DMN and MTL volume $(b=0.57,95 \%$ CI: $0.1,1.0 ; p=0.017)$ and the coefficient was only attenuated slightly with whole-brain FC in the model $(b=$ $0.54,95 \%$ CI: $\left.-4.5 \times 10^{-2}, 1.13 ; p=0.071\right)$. The magnitude of the relationship increased after controlling for $\mathrm{WMH}$, but did not reach significance in this smaller subsample $(b=0.71,95 \% \mathrm{CI}$ : $\left.-6.5 \times 10^{-2}, 1.5 ; p=0.073\right)$.

The relationship between MTL-pDMN and memory was not significant $(b=0.22,95 \% \mathrm{CI}:-0.3,0.7 p=0.436)$, even after controlling for network volumes $(b=0.3,95 \% \mathrm{CI}:-0.2,0.8 ; p=$ $0.278)$, whole-brain FC $\left(b=5.3 \times 10^{-2}, 95 \% \mathrm{CI}:-0.7,0.8 p=\right.$ $0.884)$ and $\mathrm{WMH}\left(b=1.4 \times 10^{-2}, 95 \% \mathrm{CI}:-0.9,1.0 ; p=0.977\right)$.

The specificity of the relationship between within-DMN FC and memory was assessed by examining the strength of its association with other cognitive domains. Between-person DMN FC was not associated with executive functioning ( $b=-0.6,95 \%$ CI: $-2.0,0.8 ; p=0.398)$, working memory $(b=-0.7,95 \% \mathrm{CI}$ : $-2.1,0.6 ; p=0.304)$, or processing speed $(b=1.0,95 \% \mathrm{CI}:-1.3$,
$3.4 ; p=0.385)$. Longitudinally, intraindividual changes in DMN connectivity were not significantly associated with executive functioning ( $\left.b=-7.6 \times 10^{-2}, 95 \% \mathrm{CI}:-0.8,0.6 ; p=0.828\right)$ or working memory ( $b=0.6,95 \% \mathrm{CI}:-0.1,1.36 ; p=0.099)$, but intraindividual declines in connectivity were associated with slowing of processing speed over time $(b=-1.4 ; p=0.042)$; the relationship with processing speed remained after controlling for network volume $(b=-1.44,95 \% \mathrm{CI}:-2.8,-0.05 ; p=0.042)$. Adding whole-brain FC to the model, however, reduced the association between processing speed and within-DMN FC $(b=-1.0$, 95\% CI: $-2.7,0.6 ; p=0.223)$. Adding WMHs to the model further reduced this association $(b=-0.6,95 \% \mathrm{CI}:-2.6,1.5 ; p=0.585)$.

\section{Neuropsychological performance}

To provide context to the above results, we present behavioral data describing longitudinal change in cognitive scores, including an age $\times$ time interaction to assess whether rates of change in cognition changed as a function of age.

There was a significant age $\times$ time interaction for processing speed, such the rate of slowing increased with increasing age $(b=$ $8.7 \times 10^{-3}, 95 \%$ CI: $\left.1.4 \times 10^{-3}, 1.6 \times 10^{-} 2 ; p=0.020\right)$. The age $\times$ time interactions for memory performance $(b=-2.3 \times$ $\left.10^{-3}, 95 \% \mathrm{CI}:-6.4 \times 10^{-3}, 1.7 \times 10^{-3} ; p=0.261\right)$, executive functions $\left(b=-2.3 \times 10^{-3}, 95 \% \mathrm{CI}:-6.3 \times 10^{-3} ; p=0.262\right)$, and working memory $\left(b=-2.7 \times 10^{-3}, 95 \% \mathrm{CI}:-6.9 \times 10^{-3}\right.$, $1.5 \times 10^{-3} ; p=0.210$ ) were negative but not significant, suggesting that older individuals did not decline at a significantly faster rate.

As expected, processing speed slowed over time $(b=4.4 \times$ $\left.10^{-2}, 95 \% \mathrm{CI}: 3.2 \times 10^{-3}, 8.6 \times 10^{-2} ; p=0.038\right)$. In contrast, there was a trend for improving memory performance over time $\left(b=2.3 \times 10^{-2}, 95 \% \mathrm{CI}:-3.2 \times 10^{-4}, 4.8 \times 10^{-2} ; p=0.053\right)$. No significant longitudinal changes in executive functions $\left(b=1.8 \times 10^{-2}, 95 \% \mathrm{CI}:-6.3 \times 10^{-3}, 1.8 \times 10^{-3} ; p=0.281\right)$ 
or working memory $\left(b=5.2 \times 10^{-3}, 95 \%\right.$ CI: $-1.8 \times 10^{-2}$, $\left.2.8 \times 10^{-2} ; p=0.659\right)$ were observed.

Older age was associated with slower processing speed $(b=$ $5.1 \times 10^{-2}$, $95 \%$ CI: $1.4 \times 10^{-2}, 8.8 \times 10^{-2} ; p=0.007$ ), poorer memory $\left(b=-3.1 \times 10^{-2}, 95 \%\right.$ CI: $-5.4 \times 10^{-2},-7.4 \times 10^{-3}$; $p=0.010)$, and executive functions $\left(b=-3.2 \times 10^{-2}, 95 \% \mathrm{CI}\right.$ : $\left.-5.3 \times 10^{-2},-1.0 \times 10^{-2} ; p=0.004\right)$. The relationship between age and working memory was in the expected direction but did not reach significance $\left(b=-1.6 \times 10^{-2}, 95 \%\right.$ CI: $-4.1 \times$ $\left.10^{-2}, 8.8 \times 10^{-3} ; p=0.205\right)$.

\section{Discussion}

Intraindividual trajectories of DMN and whole-brain FC were nonlinear. This interaction was non-monotic across the lifespan, such that connectivity increased over time between ages 50-70, plateaued at approximately age 70 , and declined as individuals entered older adulthood. This interaction was primarily driven by faster rates of declines in older age and was not explained by regional atrophy or WMH. Furthermore, APOE genotype was not associated with baseline connectivity, nor did genotype confer a different rate of FC change over time. Changes in cortical within-DMN FC correlated with changes in episodic memory and processing speed. Notably, neither atrophy in the cortical DMN or medial temporal network were sensitive to baseline memory performances or changes in performances over time. WMH burden and whole-brain FC did not weaken the relationship with episodic memory, suggesting a unique relationship between the DMN and memory. In contrast, the association between DMN FC and processing speed was accounted for by whole-brain changes and WMHs.

This study adds to a growing body of evidence suggesting that DMN network connectivity may demonstrate nonlinear changes across the lifespan. Although many cross-sectional studies have concluded that DMN connectivity decreases in older age, our data reveal a more complex picture. Nuances in connectivity patterns are evident when DMN subsystems are considered, and when intraindividual change is modeled rather than interpolating from cross-sectional methodology. Other studies have described nonlinear trajectories in a variety of networks (Ng et al., 2016; Ye et al., 2017), and Ng et al. (2016) reported a similar inflection point at approximately age 70 . This observation was not specific to the DMN, and our work suggests that whole-brain changes in FC should be considered before making conclusions about specific networks of the brain. Converging evidence from aging and Alzheimer's literature suggests that this nonlinearity may be driven by increased connectivity that predates or occurs concurrently with network breakdown, as well as declining connectivity in older age (Jones et al., 2011, 2016).

Although historically conceptualized as a compensatory process, several groups (O'Brien et al., 2010; Jones et al., 2016) have begun to reinterpret age-related increases in functional connectivity. These theories posit that increased synchrony of activity may reflect the inability of neurons in the DMN to handle the metabolic changes associated with aging and neuropathology, causing a shift of processing burden to downstream regions, with a short-term compensatory increase. There may be a tipping point, however, when neuropathology and the associated metabolic demands overwhelm neurons of the DMN leading to network disconnection (Hillary and Grafman, 2017). Increased or "hyperconnectivity" has been observed in a diverse range of neurologic conditions including Parkinson's disease (Stoffers et al., 2008; Simioni et al., 2016), traumatic brain injury (Hillary et al., 2014), amyotrophic lateral sclerosis (Menke et al., 2016), and frontotemporal dementia (Zhou et al., 2010; Lee et al., 2014), suggesting that increased connectivity may be a common biological response to neuropathology. In an older adult cohort such as ours, these network-level changes may be related to the accumulation of several age-related changes, including cerebrovascular disease, AD, $\alpha$-synuclein (Markesbery et al., 2009), TAR DNAbinding protein 43 (Nascimento et al., 2017), and argyrophilic grain disease (Rodriguez et al., 2016). At a cellular level, this systems-level increase in connectivity might reflect several mechanisms including amyloid-induced neuronal excitation, inflammation, dysfunction of the neurovascular unit, or prion-like spread of neurotoxic proteins. In this cohort, the nonlinear function of connectivity appeared to be independent of small vessel disease (SVD) burden, as measured by WMHs. WMHs are not sensitive or specific to all SVD. Although it is possible that this relationship was driven by neurologic changes, other potential contributors include changes in immune activation or cerebrovascular changes with normally appearing white matter. Alternatively, there may be a bias in the sample, such that those who are engaged in research have learned about healthy lifestyle behaviors and have altered their activity in a way that increases FC.

We did not find differences between $\varepsilon 4$ carriers and noncarriers in baseline or longitudinal trajectory of DMN FC. Although the APOE $\varepsilon 4$ allele confers risk for AD pathology (Corder et al., 1993), and some studies have shown that allele status affects DMN FC (Filippini et al., 2009; Zheng et al., 2017), studies in older adults have not reported a clear pattern (Sheline et al., 2010a; Machulda et al., 2011; Westlye et al., 2011; Jones et al., 2016). The null findings in the present study could be affected by power, as our group of $\varepsilon 4$ carriers was limited to 24 participants.

Similar to prior studies, we found that connectivity of the cortical DMN, including within-DMN and coupling of the anterior and posterior regions of the DMN, predicted changes in memory performance, whereas atrophy of the corresponding gray matter and hippocampal volume did not. This relationship remained above and beyond the effect of WMHs and wholebrain changes in mean connectivity, suggesting a unique role for tf-fMRI in capturing meaningful behavioral changes. It is important to note that we observed an overall trend toward improved memory performances across time, without a significant age $\times$ time interaction. This finding may indicate potential learning effects as participants perform the same memory test across testing sessions, or a "practice effect" that is not dependent on age. Nonetheless, changes in memory performance were associated with changes in DMN (both increases and decreases), suggesting there may be a physiological correlate to behavioral performances regardless of directionality.

A positive relationship between DMN FC and memory has been reported previously in cross-sectional (Vidal-Piñeiro et al., 2014) and two time point follow-up studies (Persson et al., 2014), but this provides the first truly longitudinal (i.e., 3 or more visits) support that FC in the DMN tracks memory performance in cognitively normal older adults. Although early cross-sectional work pointed to apDMN FC as the primary driver of memory performance (Andrews-Hanna et al., 2007; Vidal-Piñeiro et al., 2014), we found a larger effect for the FC between cortical DMN nodes as a whole than for apDMN connectivity. In contrast, we did not find support that changes in functional coupling of the hippocampus and posterior DMN predicted memory performance. DMN FC was also associated with processing speed, a nonspecific cognitive trait that is highly sensitive to many brain changes, including aging (Kerchner et al., 2012). Consistent with the nonspecificity of processing speed, the association between 
FC and speed was accounted for by whole-brain FC and white matter pathology.

Although FC changes correlate with visit-to-visit changes in clinical phenotype, its utility as a biomarker should be approached with caution in the context of intraindividual variability across time and interindividual variability across age. The nonlinear trajectory of DMN FC with aging introduces a challenge in using network FC as a biomarker, as the expected direction of signal change may differ depending on where an individual falls on their trajectory. This concern has been recognized and labeled pseudonormalization (Sperling et al., 2010). Additionally, visual inspection of intrasubject changes in FC was notable for substantial fluctuations. This is not unexpected given the myriad additional artifacts in BOLD signal compared with structural imaging. Despite this, the significant correlation between performance on cognitive measures and FC, but not structural imaging, suggests FC is sensitive to a unique aspect of neural functioning. The strength of the association was improved after controlling for regional atrophy, and others have shown the importance of accounting for gray matter volume (Damoiseaux et al., 2012). Multimodal imaging analyses that combine connectivity and structural imaging other imaging modalities may be a more powerful way to understand individual change and predict future decline.

This study has several limitations. The trend toward increasing connectivity in younger ages was driven by a small subsample and should be interpreted with caution. Given the large number of comparisons, our findings require validation in independent samples. Another limitation is our fairly homogeneous sample of highly educated, white participants who are not representative of many older adults in this country, and the impact of cognitive reserve in moderating the relationship between aging and $\mathrm{tf}-$ fMRI deserves empirical evaluation. This study is susceptible to common confounds associated with longitudinal designs, including practice effects and regression to the mean. Finally, several processing decisions we made could affect the outcomes, including our method of defining networks and our decision not to regress out global signal. Based on recent work suggesting unintended removal of true neural connectivity when controlling for motion at the group level (Geerligs et al., 2017), we did not covary for motion beyond the nuisance covariates used at the level of matrix calculation.

Whole-brain and DMN FC is sensitive to the aging process, and within-DMN FC is a specific marker of changes in memory performance. Particularly interesting is the potential FC increases in some younger participants, which may reflect a broad range of neural, vascular, and metabolic changes that occur with aging. The present work also highlights the importance of gathering longitudinal data to capture nonlinear and potentially nonmonotonic relationships between aging and the brain's intrinsic connectivity. Functional connectivity may serve as a biomarker of clinical change by capturing system-level network alterations caused by early cellular dysfunction that is not easily quantified using standard volumetric imaging.

\section{References}

Andrews-Hanna JR, Snyder AZ, Vincent JL, Lustig C, Head D, Raichle ME, Buckner RL (2007) Disruption of large-scale brain systems in advanced aging. Neuron 56:924-935. CrossRef Medline

Andrews-Hanna JR, Reidler JS, Sepulcre J, Poulin R, Buckner RL (2010) Functional-anatomic fractionation of the brain's default network. Neuron 65:550-562. CrossRef Medline

Ashburner J (2007) A fast diffeomorphic image registration algorithm. Neuroimage 38:95-113. CrossRef Medline
Ashburner J, Friston KJ (2005) Unified segmentation. Neuroimage 26:839851. CrossRef Medline

Ashburner J, Ridgway GR (2012) Symmetric diffeomorphic modeling of longitudinal structural MRI. Front Neurosci 6:197. CrossRef Medline

Avants BB, Tustison NJ, Wu J, Cook PA, Gee JC (2011) An open source multivariate framework for $n$-tissue segmentation with evaluation on public data. Neuroinformatics 9:381-400. CrossRef Medline

Barkhof F, Haller S, Rombouts SA (2014) Resting-state functional MR imaging: a new window to the brain. Radiology 272:29-49. CrossRef Medline

Bernard C, Dilharreguy B, Helmer C, Chanraud S, Amieva H, Dartigues JF, Allard M, Catheline G (2015) PCC characteristics at rest in 10-year memory decliners. Neurobiol Aging 36:2812-2820. CrossRef Medline

Braak H, Del Tredici K (2012) Where, when, and in what form does sporadic Alzheimer's disease begin? Curr Opin Neurol 25:708-714. CrossRef Medline

Corder EH, Saunders AM, Strittmatter WJ, Schmechel DE, Gaskell PC, Small GW, Roses AD, Haines JL, Pericak-Vance MA (1993) Gene dose of apolipoprotein E type 4 allele and the risk of Alzheimer's disease in late onset families. Science 261:921-923. CrossRef Medline

Dadar M, Pascoal TA, Manitsirikul S, Misquitta K, Fonov VS, Tartaglia MC, Breitner J, Rosa-Neto P, Carmichael OT, Decarli C, Collins DL (2017) Validation of a regression technique for segmentation of white matter hyperintensities in Alzheimer's disease. IEEE Trans Med Imaging 36: 1758-1768. CrossRef Medline

Damoiseaux JS, Beckmann CF, Arigita EJ, Barkhof F, Scheltens P, Stam CJ, Smith SM, Rombouts SA (2008) Reduced resting-state brain activity in the "default network" in normal aging. Cereb Cortex 18:1856-1864. CrossRef Medline

Damoiseaux JS, Prater KE, Miller BL, Greicius MD (2012) Functional connectivity tracks clinical deterioration in Alzheimer's disease. Neurobiol Aging 33:828.e19-828.e30. CrossRef Medline

Delis DC, Kramer JH, Kaplan E, Ober BA (2000) California verbal learning test, Ed 2, adult version: manual. San Antonio, TX: Psychological Corporation.

Delis DC, Kaplan E, Kramer JH (2001) Delis-Kaplan executive function system (DKEFS): examiner's manual. San Antonio, TX: Psychological Corporation.

Fan L, Li H, Zhuo J, Zhang Y, Wang J, Chen L, Yang Z, Chu C, Xie S, Laird AR, Fox PT, Eickhoff SB, Yu C, Jiang T (2016) The human brainnetome atlas: a new brain atlas based on connectional architecture. Cereb Cortex 26:3508-3526. CrossRef Medline

Filippini N, MacIntosh BJ, Hough MG, Goodwin GM, Frisoni GB, Smith SM, Matthews PM, Beckmann CF, Mackay CE (2009) Distinct patterns of brain activity in young carriers of the APOE-epsilon4 allele. Proc Natl Acad Sci U S A 106:7209-7214. CrossRef Medline

Fjell AM, Sneve MH, Grydeland H, Storsve AB, de Lange AG, Amlien IK, Røgeberg OJ, Walhovd KB (2015) Functional connectivity change across multiple cortical networks relates to episodic memory changes in aging. Neurobiol Aging 36:3255-3268. CrossRef Medline

Geerligs L, Tsvetanov KA, Cam-CAN RN, Henson RN (2017) Challenges in measuring individual differences in functional connectivity using fMRI: the case of healthy aging. Hum Brain Mapp 38:4125-4156. CrossRef Medline

Greicius MD, Srivastava G, Reiss AL, Menon V (2004) Default-mode network activity distinguishes Alzheimer's disease from healthy aging: evidence from functional MRI. Proc Natl Acad Sci U S A 101:4637-4642. CrossRef Medline

Hedden T, Van Dijk KR, Becker JA, Mehta A, Sperling RA, Johnson KA, Buckner RL (2009) Disruption of functional connectivity in clinically normal older adults harboring amyloid burden. J Neurosci 29:1268612694. CrossRef Medline

Hillary FG, Grafman JH (2017) Injured brains and adaptive networks: the benefits and costs of hyperconnectivity. Trends Cogn Sci 21:385-401. CrossRef Medline

Hillary FG, Rajtmajer SM, Roman CA, Medaglia JD, Slocomb-Dluzen JE, Calhoun VD, Good DC, Wylie GR (2014) The rich get richer: brain injury elicits hyperconnectivity in core subnetworks. PLoS One 9:e104021. CrossRef Medline

Jones DT, Machulda MM, Vemuri P, McDade EM, Zeng G, Senjem ML, Gunter JL, Przybelski SA, Avula RT, Knopman DS, Boeve BF, Petersen RC, Jack CR Jr (2011) Age-related changes in the default mode network 
are more advanced in alzheimer disease. Neurology 77:1524-1531. CrossRef Medline

Jones DT, Knopman DS, Gunter JL, Graff-Radford J, Vemuri P, Boeve BF, Petersen RC, Weiner MW, Jack CR Jr (2016) Cascading network failure across the Alzheimer's disease spectrum. Brain 139:547-562. CrossRef Medline

Kerchner GA, Racine CA, Hale S, Wilheim R, Laluz V, Miller BL, Kramer JH (2012) Cognitive processing speed in older adults: relationship with white matter integrity. PLoS One 7:e50425. CrossRef Medline

Kramer JH, Jurik J, Sha SJ, Rankin KP, Rosen HJ, Johnson JK, Miller BL (2003) Distinctive neuropsychological patterns in frontotemporal dementia, semantic dementia, and Alzheimer disease. Cogn Behav Neurol 16:211-218. CrossRef Medline

Kramer JH, Mungas D, Possin KL, Rankin KP, Boxer AL, Rosen HJ, Bostrom A, Sinha L, Berhel A, Widmeyer M (2014) NIH EXAMINER: conceptualization and development of an executive function battery. J Int Neuropsychol Soc 20:11-19. CrossRef Medline

Lee SE, Khazenzon AM, Trujillo AJ, Guo CC, Yokoyama JS, Sha SJ, Takada LT, Karydas AM, Block NR, Coppola G, Pribadi M, Geschwind DH, Rademakers R, Fong JC, Weiner MW, Boxer AL, Kramer JH, Rosen HJ, Miller BL, Seeley WW, et al. (2014) Altered network connectivity in frontotemporal dementia with C9orf72 hexanucleotide repeat expansion. Brain 137:3047-3060. CrossRef Medline

Li K, Laird AR, Price LR, McKay DR, Blangero J, Glahn DC, Fox PT (2016) Progressive bidirectional age-related changes in default mode network effective connectivity across six decades. Front Aging Neurosci 8:137. CrossRef Medline

Machulda MM, Jones DT, Vemuri P, McDade E, Avula R, Przybelski S, Boeve BF, Knopman DS, Petersen RC, Jack CR Jr (2011) Effect of APOE $\varepsilon 4$ status on intrinsic network connectivity in cognitively normal elderly subjects. Arch Neurol 68:1131-1136. CrossRef Medline

Markesbery WR, Jicha GA, Liu H, Schmitt FA (2009) Lewy body pathology in normal elderly subjects. J Neuropathol Exp Neurol 68:816-822. CrossRef Medline

Mazziotta J, Toga A, Evans A, Fox P, Lancaster J, Zilles K, Woods R, Paus T, Simpson G, Pike B, Holmes C, Collins L, Thompson P, MacDonald D, Iacoboni M, Schormann T, Amunts K, Palomero-Gallagher N, Geyer S, Parsons L, et al. (2001) A four-dimensional probabilistic atlas of the human brain. J Am Med Inform Assoc 8:401-430. CrossRef Medline

Menke RA, Proudfoot M, Wuu J, Andersen PM, Talbot K, Benatar M, Turner MR (2016) Increased functional connectivity common to symptomatic amyotrophic lateral sclerosis and those at genetic risk. J Neurol Neurosurg Psychiatry 87:580-588. CrossRef Medline

Nascimento C, Di Lorenzo Alho AT, Bazan Conceição Amaral C, Leite REP, Nitrini R, Jacob-Filho W, Pasqualucci CA, Kastehelmi Hokkanen SR, Hunter S, Keage H, Kovacs GG, Grinberg LT, Suemoto CK (2017) Prevalence of TDP-43 proteinopathy in cognitively normal older adults: systematic review and meta-analysis. Neuropathol Appl Neurobiol. Advance online publication. doi: 10.1111/nan.12430.

Ng KK, Lo JC, Lim JKW, Chee MWL, Zhou J (2016) Reduced functional segregation between the default mode network and the executive control network in healthy older adults: a longitudinal study. Neuroimage 133: 321-330. CrossRef Medline

O’Brien JL, O'Keefe KM, LaViolette PS, DeLuca AN, Blacker D, Dickerson BC, Sperling RA (2010) Longitudinal fMRI in elderly reveals loss of hippocampal activation with clinical decline. Neurology 74:1969-1976. CrossRef Medline

Persson J, Pudas S, Nilsson LG, Nyberg L (2014) Longitudinal assessment of default-mode brain function in aging. Neurobiol Aging 35:2107-2117. CrossRef Medline

Power JD, Cohen AL, Nelson SM, Wig GS, Barnes KA, Church JA, Vogel AC, Laumann TO, Miezin FM, Schlaggar BL, Petersen SE (2011) Functional network organization of the human brain. Neuron 72:665-678. CrossRef Medline

Rodriguez RD, Suemoto CK, Molina M, Nascimento CF, Leite RE, de Lucena Ferretti-Rebustini RE, Farfel JM, Heinsen H, Nitrini R, Ueda K, Pasqualucci CA, Jacob-Filho W, Yaffe K, Grinberg LT (2016) Argyrophilic grain disease: demographics, clinical, and neuropathological features from a large autopsy study. J Neuropathol Exp Neurol 75:628-635. CrossRef Medline

Rubinov, M, Sporns, O (2011) Weight-conserving characterization of complex functional brain networks. Neuroimage 56:2068-2079. CrossRef

Sala-Llonch R, Bartrés-Faz D, Junqué C (2015) Reorganization of brain networks in aging: a review of functional connectivity studies. Front Psychol 6:663. CrossRef Medline

Satterthwaite TD, Elliott MA, Gerraty RT, Ruparel K, Loughead J, Calkins ME, Eickhoff SB, Hakonarson H, Gur RC, Gur RE, Wolf DH (2013) An improved framework for confound regression and filtering for control of motion artifact in the preprocessing of resting-state functional connectivity data. Neuroimage 64:240-256. CrossRef Medline

Sheline YI, Morris JC, Snyder AZ, Price JL, Yan Z, D'Angelo G, Liu C, Dixit S, Benzinger T, Fagan A, Goate A, Mintun MA (2010a) APOE4 allele disrupts resting state $\mathrm{PMRI}$ connectivity in the absence of amyloid plaques or decreased CSF A 342 . J Neurosci 30:17035-17040. CrossRef Medline

Sheline YI, Raichle ME, Snyder AZ, Morris JC, Head D, Wang S, Mintun MA (2010b) Amyloid plaques disrupt resting state default mode network connectivity in cognitively normal elderly. Biol Psychiatry 67:584-587. CrossRef Medline

Simioni AC, Dagher A, Fellows LK (2016) Compensatory striatal-cerebellar connectivity in mild-moderate Parkinson's disease. Neuroimage Clin 10: 54-62. CrossRef Medline

Sperling RA, Dickerson BC, Pihlajamaki M, Vannini P, LaViolette PS, Vitolo OV, Hedden T, Becker JA, Rentz DM, Selkoe DJ, Johnson KA (2010) Functional alterations in memory networks in early Alzheimer's disease. NeuroMolecular Med 12:27-43. CrossRef Medline

StataCorp (2015) Stata statistical software: release 14.

Stoffers D, Bosboom JL, Deijen JB, Wolters EC, Stam CJ, Berendse HW (2008) Increased cortico-cortical functional connectivity in early-stage Parkinson's disease: an MEG study. Neuroimage 41:212-222. CrossRef Medline

Stroop JR (1935) Studies of interference in serial verbal reactions. J Exp Psychol 18:643-662. CrossRef

Su YY, Zhang XD, Schoepf UJ, Varga-Szemes A, Stubenrauch A, Liang X, Zheng LJ, Zheng G, Kong X, Xu Q, Wang SJ, Qi RF, Lu GM, Zhang LJ (2017) Lower functional connectivity of default mode network in cognitively normal young adults with mutation of APP, presenilins and APOE $\varepsilon 4$. Brain Imaging Behav 11:818-828. CrossRef Medline

Vidal-Piñeiro D, Valls-Pedret C, Fernández-Cabello S, Arenaza-Urquijo EM, Sala-Llonch R, Solana E, Bargalló N, Junqué C, Ros E, Bartrés-Faz D (2014) Decreased default mode network connectivity correlates with age-associated structural and cognitive changes. Front Aging Neurosci 6:256. CrossRef Medline

Ward AM, Mormino EC, Huijbers W, Schultz AP, Hedden T, Sperling RA (2015) Relationships between default-mode network connectivity, medial temporal lobe structure, and age-related memory deficits. Neurobiol Aging 36:265-272. CrossRef Medline

Wechsler D (1997) Wechsler adult intelligence scale. San Antonio, TX: Psychological Corporation.

Westlye ET, Lundervold A, Rootwelt H, Lundervold AJ, Westlye LT (2011) Increased hippocampal default mode synchronization during rest in middle-aged and elderly APOE $\varepsilon 4$ carriers: relationships with memory performance. J Neurosci 31:7775-7783. CrossRef Medline

Ye Q, Su F, Shu H, Gong L, Xie C, Zhang Z, Bai F (2017) The apolipoprotein E gene affects the three-year trajectories of compensatory neural processes in the left-lateralized hippocampal network. Brain Imaging Behav 11: 1446-1458. CrossRef Medline

Zheng LJ, Su YY, Wang YF, Schoepf UJ, Varga-Szemes A, Pannell J, Liang X, Zheng G, Lu GM, Yang GF, Zhang LJ (2017) Different hippocampus functional connectivity patterns in healthy young adults with mutations of APP/presenilin-1/2 and APOE $\varepsilon 4$. Mol Neurobiol. Advance online publication. Retrieved July 18, 2017. CrossRef Medline

Zhou J, Greicius MD, Gennatas ED, Growdon ME, Jang JY, Rabinovici GD, Kramer JH, Weiner M, Miller BL, Seeley WW (2010) Divergent network connectivity changes in behavioural variant frontotemporal dementia and Alzheimer's disease. Brain 133:1352-1367. CrossRef Medline 